Research Article

\title{
Identification of PDL1-Related Biomarkers to Select Lung Adenocarcinoma Patients for PD1/PDL1 Inhibitors
}

\author{
Yanping Wu $(\mathbb{D}$, Lianjun Lin $(\mathbb{D}$, and Xinmin Liu $(\mathbb{D})$ \\ Department of Geriatrics, Peking University First Hospital, Beijing 100034, China \\ Correspondence should be addressed to Xinmin Liu; lxm2128@163.com
}

Received 15 October 2019; Revised 15 March 2020; Accepted 25 May 2020; Published 10 June 2020

Academic Editor: Chiara Nicolazzo

Copyright (C) 2020 Yanping Wu et al. This is an open access article distributed under the Creative Commons Attribution License, which permits unrestricted use, distribution, and reproduction in any medium, provided the original work is properly cited.

\begin{abstract}
PD1/PDL1 inhibitors have been adopted for the treatment of advanced non-small-cell lung cancer, and PDL1 expression has been investigated as a predictive biomarker for PD1/PDL1 inhibitor therapy. However, PDL1 lacks diagnostic accuracy in differentiating patients who are likely or unlikely to benefit. So, it is urgent and clinically significant to identify other associated predictive biomarkers for PD1/PDL1 inhibitor therapy. Our work was to identify PDL1-related biomarkers that could improve the patient selection for PD1/PDL1 inhibitor treatment. We obtained 500 genes coexpressed with PDL1 in lung adenocarcinoma from the TCGA database. Then, we identified 125 out of 500 genes differentially expressed in lung adenocarcinoma. A total of 39 genes were distinguished with prognostic value and associated with overall survival. Median survival time analysis based on gene expression level, protein-protein interaction analysis, GO and KEGG enrichment analyses, and significant GO and KEGG function consistency analyses were conducted to screen candidate biomarkers. Three candidate genes, BRCA1, BRIP1, and EREG, were identified to be functionally significantly coexpressed with PDL1. Functional enrichment analysis and protein-protein interaction networks further showed that these genes mainly participated in immune response and cell activation. Additionally, to find potential adjuvant therapeutic targets in PD1/PDL1 inhibitor treatment, we performed transcription factor prediction analysis. A group of negative differential expression but PDL1-related biomarkers has been identified, which might help to assess the clinical management of lung cancer patients. A combination of potential biomarkers and adjuvant therapeutic targets with PDL1 will predict the response to PD1/PDL1 inhibitors more accurately and help with the patient selection for more personalized immune checkpoint inhibitor treatment.
\end{abstract}

\section{Introduction}

Lung cancer is a leading cause of cancer death worldwide due to its low survival rate $[1,2]$. Non-small-cell lung cancer (NSCLC) accounts for $85 \%$ of lung cancer, and lung adenocarcinoma is the most common histological type of NSCLC [3]. Traditional therapeutic options remain limited for patients, and recently, immunotherapy emerges and becomes popular because of its outstanding efficacy [4]. PD1/PDL1 immune checkpoint inhibitors have been developed and adopted for the treatment of NSCLC. PD1 is expressed by activated T cells, B lymphocytes, and natural killer cells. PDL1 was identified as PD1 ligand. PDL1 is expressed by T lymphocytes, epithelial cells, endothelial cells, tumor cells, and other cells in the local tumor environment. The interac- tion between PD1 and PDL1 suppresses T cell activation and helps tumor cells to escape immune surveillance.

Nowadays PD1/PDL1 immune checkpoint inhibitors, including nivolumab, pembrolizumab, atezolizumab, and durvalumab, have been adopted to treat NSCLC $[5,6]$. A study revealed that pembrolizumab monotherapy has shown significant improvements in overall survival as a first-line treatment compared with conventional chemotherapy for locally advanced or metastatic non-small-cell lung cancer without sensitising EGFR or ALK alterations when PDL1 TPS $\geq 1 \%$ [7]. Pembrolizumab monotherapy now is recommended as a first-line therapy for patients with NSCLC without driver gene mutations and with a high PDL1 expression (tumor proportion score (TPS) $\geq 50 \%$ ). Nivolumab or atezolizumab is recommended in a second-line setting for NSCLC 
regardless of PDL1 expression. A multicentre, randomised, open-label, phase 3 trial revealed that the treatment benefit was observed in terms of overall survival and progressionfree survival in the subgroup populations when atezolizumab in combination with carboplatin plus nab-paclitaxel chemotherapy as the first-line treatment for metastatic nonsquamous non-small-cell lung cancer, regardless of PDL1 expression [8]. Atezolizumab is now FDA approved in the first-line setting in combination with carboplatin, paclitaxel, and bevacizumab for patients with metastatic nonsquamous NSCLC with no EGFR or ALK genomic tumor mutations. Regarding locally advanced NSCLC, durvalumab currently represents the only FDA-approved and recommended immune checkpoint inhibitor for the treatment of unresectable stage III NSCLC patients, irrespective of histological type and PDL1 expression, whose disease has not progressed after a previous chemoradiotherapy treatment [9]. However, according to a recent meta-analysis, among patients with PDL1 expression $<1 \%$, docetaxel monotherapy second-line treatment was not superior to immune checkpoint inhibitors [10].

At present, PDL1 is the only predictive biomarker validated for the selection of patients who could benefit from pembrolizumab, and PDL1 expression in tumor cells is considered to be prognostic in NSCLC. However, so far, there is no consensus in defining the PDL1 expression level as positive or negative (ranging from $1 \%$ to $50 \%$ expression) [11]. And there is significant intratumor heterogeneity for the PDL1 expression, and a biopsy may not be representative of the entire tumor mass [12]. Another debate focus is whether immunotherapy is active in patients with NSCLC who have an activating genetic abnormality such as EGFR mutation or ALK translocation. Then, the inevitable drug resistance issue and the mechanisms of resistance are currently poorly understood. Given the controversial results and important drawbacks, it is important to find other potential, reliable biomarkers in combination with PDL1 to improve the selection of patients for PD1/PDL1 inhibitor treatment.

\section{Materials and Methods}

2.1. Patients and Samples. The mRNA sequencing data and related clinical information of 533 lung adenocarcinoma tissue samples and 59 adjacent nontumor tissue samples were obtained from The Cancer Genome Atlas (TCGA) database. Patients who had other malignancies than lung adenocarcinoma were excluded. As the data were retrieved from TCGA database, data processing procedures in this study met the guideline of the TCGA human subject protection and data access policies.

2.2. Differential Expression Analysis. We performed differential expression analysis by comparing mRNA expression in human lung adenocarcinoma tissues and adjacent normal tissues. The differential expression of mRNAs between human lung adenocarcinoma tissues and adjacent nontumor tissues were compared using the DESeq2 $\mathrm{R}$ package with Wald significance tests. The DESeq 2 model and all the steps taken in the software were described in a previous publica- tion [13], and we include the formula and descriptions in this section as well. The differential expression analysis in DESeq2 uses a generalized linear model of the form:

$$
\begin{gathered}
K_{i j} \sim \operatorname{NB}\left(\mu_{i j}, \alpha_{i}\right), \\
\mu_{i j}=s_{j} q_{i j}, \\
\log _{2}\left(q_{i j}\right)=x_{j}, \beta_{i},
\end{gathered}
$$

where counts $K_{i j}$ for gene $i$, sample $j$ are modeled using a negative binomial distribution with fitted mean $\mu_{i j}$ and a genespecific dispersion parameter $\alpha_{i}$. The fitted mean is composed of a sample-specific size factor $s_{j}$ and a parameter $q_{i j}$ proportional to the expected true concentration of fragments for sample $j$. The coefficients $\beta_{i}$ give the $\log _{2}$ fold changes for gene $i$ for each column of the model matrix $x$. Note that the model can be generalized to use sample- and genedependent normalization factors $s_{i j} . P$ values less than 0.05 were considered statistically significant.

2.3. Coexpression Analysis. We generated mRNAs-PDL1 coexpression network based on the human lung adenocarcinoma-associated genes in TCGA by the cor function of R package. Significant correlation pairs were used to construct the network based on the Pearson correlation coefficients and the $P$ values. The annotation of protein cellular localization and biological function was performed by using the protein-protein interaction (PPI) network. The PPI network was retrieved from the STRING database and reconstructed via Cytoscape software. To assess the main function of the PDL1-associated genes, Gene Ontology (GO) and Kyoto Encyclopedia of Genes and Genomes (KEGG) analyses were performed using the ClusterProfile of $\mathrm{R}$ package with the hypergeometric distribution test.

$$
p=1-\sum_{i=0}^{k-1} \frac{\left(\begin{array}{c}
M \\
i
\end{array}\right)\left(\begin{array}{c}
N-M \\
n-i
\end{array}\right)}{\left(\begin{array}{l}
N \\
n
\end{array}\right)} .
$$

In this equation, $N$ is the total number of genes in background distribution, $M$ is the number of genes within that distribution that are annotated (either directly or indirectly) to the gene set of interest, $n$ is the size of the list of genes of interest, and $k$ is the number of genes within that list which are annotated to the gene set. $P$ value $<0.05$ was set as the cutoff criterion.

2.4. Survival Analysis. We performed survival analysis to further investigate whether the coexpressed genes were associated with the prognosis. Genes were divided into two groups according to the median of gene expression. The gene expression was labeled as high or low using the dichotomy method to determine which gene could potentially be of functional significance in lung adenocarcinoma prognosis. Univariate Cox regression analysis was performed to identify 


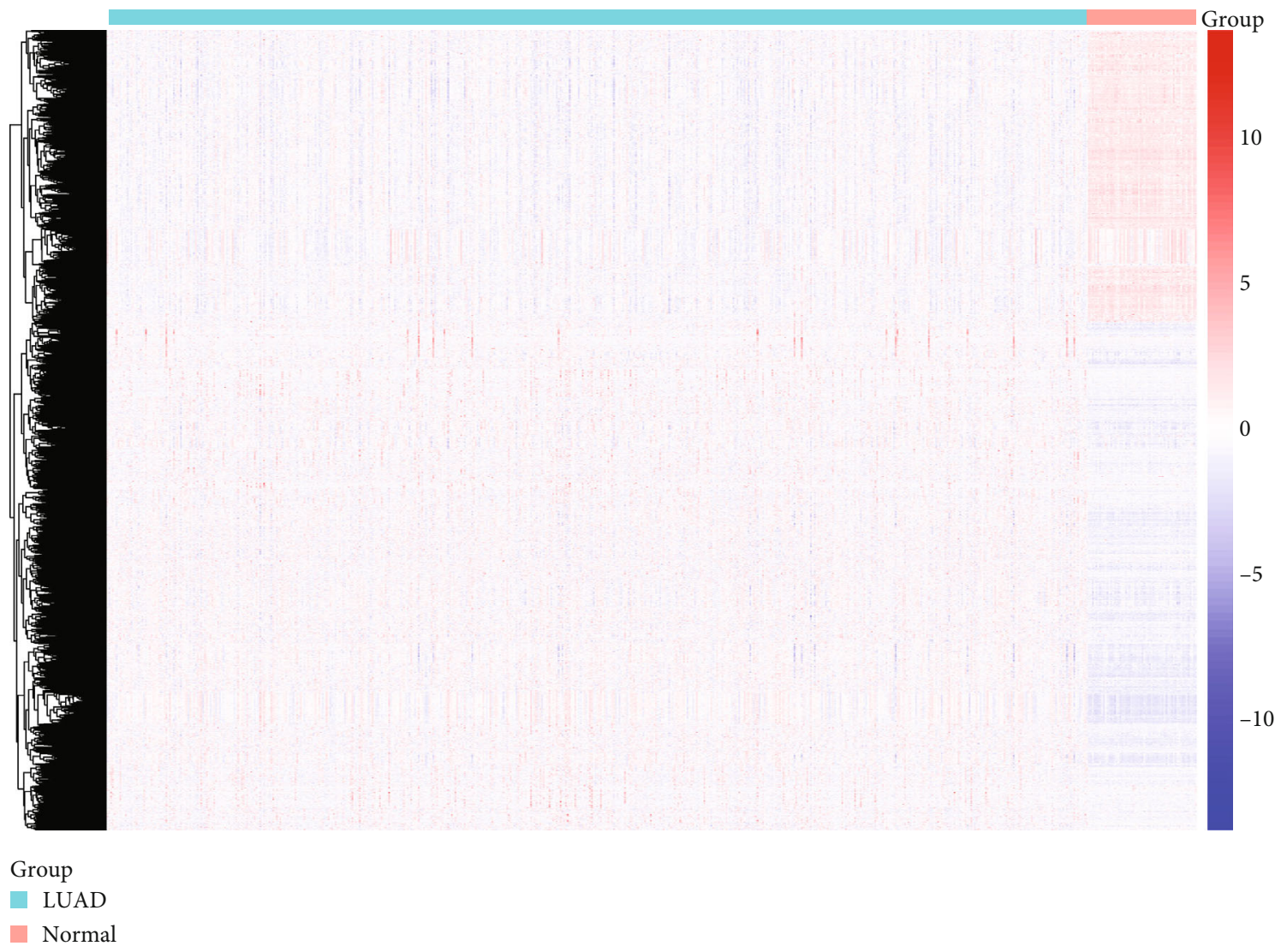

Figure 1: Differentially expressed genes in lung adenocarcinoma. A heat map is showing the differentially expressed mRNAs.

survival-related genes. Kaplan-Meier survival analysis with a log-rank test was performed to compare the differences in overall survival between the high-expression group and low-expression group. The survival curve is calculated by survival $\mathrm{R}$ package and plotted by surfminer. The survival probability is calculated by the survival time, survival state, and different grouping conditions, as follows:

$$
\widehat{S}_{K M}(t)=\prod_{s<t} \frac{\bar{Y}(t s)-t(s)}{\bar{Y}(s)}
$$

Graphically, the Kaplan-Meier survival curve appears as a step function with a drop at each death. Censoring times are often marked on the plot as $\backslash+$ " symbols. KM curves are created with the survfit function. The left-hand side of the formula will be a Surv object and the right-hand side contains one or more categorical variables that will divide the observations into groups.

2.5. Transcription Factor Binding Site Prediction. We performed transcription factor binding site (TFBS) prediction analysis of the interest genes. Besides, published data of genes and their transcription factors were collected, we also used http://www.tfbss.org/search/ method to predict the transcription factors of target genes.

\section{Results}

3.1. Identification of Differentially Expressed mRNAs in Human Lung Adenocarcinoma. To identify significantly differentially expressed mRNAs, we initially performed mRNA differential expression analysis in lung adenocarcinoma and adjacent normal tissues. A total of 533 lung adenocarcinoma samples and 59 normal samples were obtained from TCGA database. We set fold change $>2$ and $P$ value $<0.05$ as cutoffs to screen significantly differentially expressed mRNAs. Ultimately, a total of 5439 differentially expressed mRNAs were identified, as shown in Figure 1. Among the differentially expressed genes, 3456 genes were upregulated and the rest of the genes were downregulated.

3.2. Coexpression of Lung Adenocarcinoma Genes and PDL1. To identify coexpressed genes, we assessed the correlation between PDL1 and lung adenocarcinoma-associated genes in TCGA database. We obtained the top 500 genes when the Pearson correlation coefficient $>0.308571756$ and $<-0.309493137$. Scatter plot showing the coexpression pattern of each lung adenocarcinoma gene and PD-L1 was conducted. Among those genes, when $P$ values $<0.05$ and fold change $>2$ as cutoffs, 125 genes were identified not only coexpressed with PDL1 but also significantly differentially expressed in lung adenocarcinoma. To find out whether those genes identified from TCGA database are also of prognostic significance, we generated the Kaplan-Meier survival 
curves to explore the potential roles of those genes in overall survival. Among the candidate genes identified in lung adenocarcinoma, a total of 39 genes were validated having a significant effect on the overall survival of patients in the logrank test $(P<0.05$, all genes were listed in Table 1 , two were approximately equal to 0.05 and were excluded as candidates in the following analysis). To assess the prognostic value of the identified biomarkers (by median), 6 genes were excluded according to the survival time two more weeks less than that of PDL1 as a biomarker. To better understand the interplay among the identified coexpressed genes, we obtained the protein-protein interaction (PPI) network using the STRING tool, as shown in Figure 2. Nine genes that not interacted with others were excluded.

3.3. Function Assessment. Furthermore, to understand the biological functions and processes these genes were involved in, GO and KEGG enrichment analyses were conducted. Most of the PDL1-associated mRNAs in the coexpression network could be assigned to functional classes related to immune functions and cancer-related pathways. This process revealed enrichment of 45 KEGG pathways $(P$ value $<0.05$ and enrichment score $>2)$ and 402 GO terms $(P$ value < 0.05 and enrichment score $>10)$. Five of the top 15 pathways were immune-related pathways including natural killer cellmediated cytotoxicity, Toll-like receptor signaling pathway, $B$ cell receptor signaling pathway, PDL1 expression and PD1 checkpoint pathway in cancer, and T cell receptor signaling pathway. Top GO terms were Toll-like receptor 2 signaling pathway, positive regulation of gamma-delta $\mathrm{T}$ cell activation, cellular response to interferon-beta, regulation of response to tumor cell, and regulation of immune response to tumor cell. Go and KEGG enrichment analyses of 39 candidate genes was shown in Figure 3. Six genes, BRCA1, BRIP1, CSF2RB, CYBB, and EREG, TLR4, were identified according to the consistency of significant GO function and signaling pathway. Eventually, BRCA1, BRIP1, and EREG were chosen as the candidates based on the clinical research progress through searching the PubMed database. Median survival time of patients in the PDL1 high-expression group was 701 days. Median survival time of the identified biomarkers, BRCA1, BRIP1, and EREG, were 690, 701, and 690 days separately. Kaplan-Meier survival curve of three candidate genes was shown in Figure 4.

3.4. Possible Adjuvant Therapeutic Targets Based on Transcription Factor Prediction. To identify the possible adjuvant therapeutic targets based on transcription factor prediction, we performed transcription factor prediction analysis. Transcription factor prediction analysis was applied to 152 significantly overlapped genes that were recognized by GO and KEGG enrichment intersection analyses. Statistically significant top 10 predictions of EREG, BRCA1, and BRIP1 were listed, as shown in Table 2.

3.5. Possible Adjuvant Biomarkers for Patient Selection Based on Nondifferentially Expressed Genes. To identify other possible adjuvant biomarkers for patient selection, we focus on the 5476 nondifferentially expressed genes. Among the top 500
TABLE 1: PDL1 coexpressed genes not only differentially expressed in lung adenocarcinoma but also contribute to overall survival.

\begin{tabular}{|c|c|c|c|}
\hline mRNA & Cor & $\log _{2}$ foldchange & $P$ value \\
\hline ANLN & 0.36109 & 3.77289 & 5.92E-05 \\
\hline ARNTL2 & 0.3383 & 2.4906 & 0.00144954 \\
\hline AVL9 & 0.31527 & 1.08517 & 0.04576124 \\
\hline BRCA1 & 0.3755 & 1.46278 & 0.005052667 \\
\hline BRIP1 & 0.32595 & 2.68693 & 0.04478685 \\
\hline CD53 & 0.37628 & -1.0313 & 0.005429641 \\
\hline CENPE & 0.32484 & 2.65322 & 0.006552998 \\
\hline CRTAM & 0.37113 & -1.0706 & 0.02319598 \\
\hline CSF2RB & 0.32911 & -1.0044 & 0.008033928 \\
\hline CYBB & 0.46179 & -1.3471 & 0.01465277 \\
\hline DOCK11 & 0.42634 & -1.2654 & 0.01724113 \\
\hline ELOVL6 & 0.41838 & 1.28021 & 0.01821207 \\
\hline EREG & 0.51626 & 2.58762 & 0.003137182 \\
\hline FCGR1B & 0.346 & -1.0663 & 0.01076134 \\
\hline GIMAP4 & 0.37656 & -1.4853 & 0.001781643 \\
\hline GIMAP5 & 0.34985 & -1.6241 & 0.006549764 \\
\hline GPR65 & 0.37454 & -1.1182 & 0.03443263 \\
\hline GPX3 & 0.34227 & -2.2637 & 0.009965236 \\
\hline GSG2 & 0.31185 & 1.92806 & 0.04657218 \\
\hline HACD4 & 0.37455 & -1.4402 & 0.002306662 \\
\hline KIAA1524 & 0.33152 & 1.96877 & 0.01320607 \\
\hline KIF20B & 0.34274 & 1.29505 & 0.02865572 \\
\hline KRT80 & 0.32673 & 2.43778 & 0.01169369 \\
\hline LPXN & 0.33821 & -1.1081 & 0.004965741 \\
\hline LRRC8C & 0.33574 & -1.12 & 0.0407523 \\
\hline MELK & 0.35309 & 3.61062 & 0.01053599 \\
\hline MNDA & 0.35278 & -1.4709 & 0.02036211 \\
\hline NCAPG2 & 0.31191 & 1.77961 & 0.003450744 \\
\hline NCKAP1L & 0.37919 & -1.2778 & 0.003063421 \\
\hline NGEF & 0.31071 & 3.00327 & 0.01781168 \\
\hline PLK4 & 0.32713 & 2.03832 & 0.01624677 \\
\hline PMAIP1 & 0.40395 & 1.63802 & 0.03528504 \\
\hline RALGPS2 & 0.31509 & 1.65783 & 0.02929378 \\
\hline RGS20 & 0.35033 & 2.9063 & 0.009297155 \\
\hline SCIMP & 0.31898 & -1.5645 & 0.000474133 \\
\hline SHCBP1 & 0.42305 & 2.27346 & 0.001114681 \\
\hline SMCO2 & 0.33114 & 2.49326 & 0.002347819 \\
\hline TLR4 & 0.35033 & -1.5721 & 0.0217862 \\
\hline TRIM6 & 0.31406 & 1.16306 & 0.01236621 \\
\hline
\end{tabular}

Cor: correlation coefficient; $\log _{2}$ foldchange: negative number represents downregulation, positive number represents upregulation; $P$ value: overall survival of patients in log-rank test.

PDL1-correlated genes, only 114 genes have a negative differential expression in cancer tissues compared with normal tissues. The number of genes that correlated with the overall survival was 21 . Eventually, 4 genes, CCR5, FOSL1, NAIP, and NBN, stand out depending to the consistency of significant GO function and signaling pathway and PPI network. 


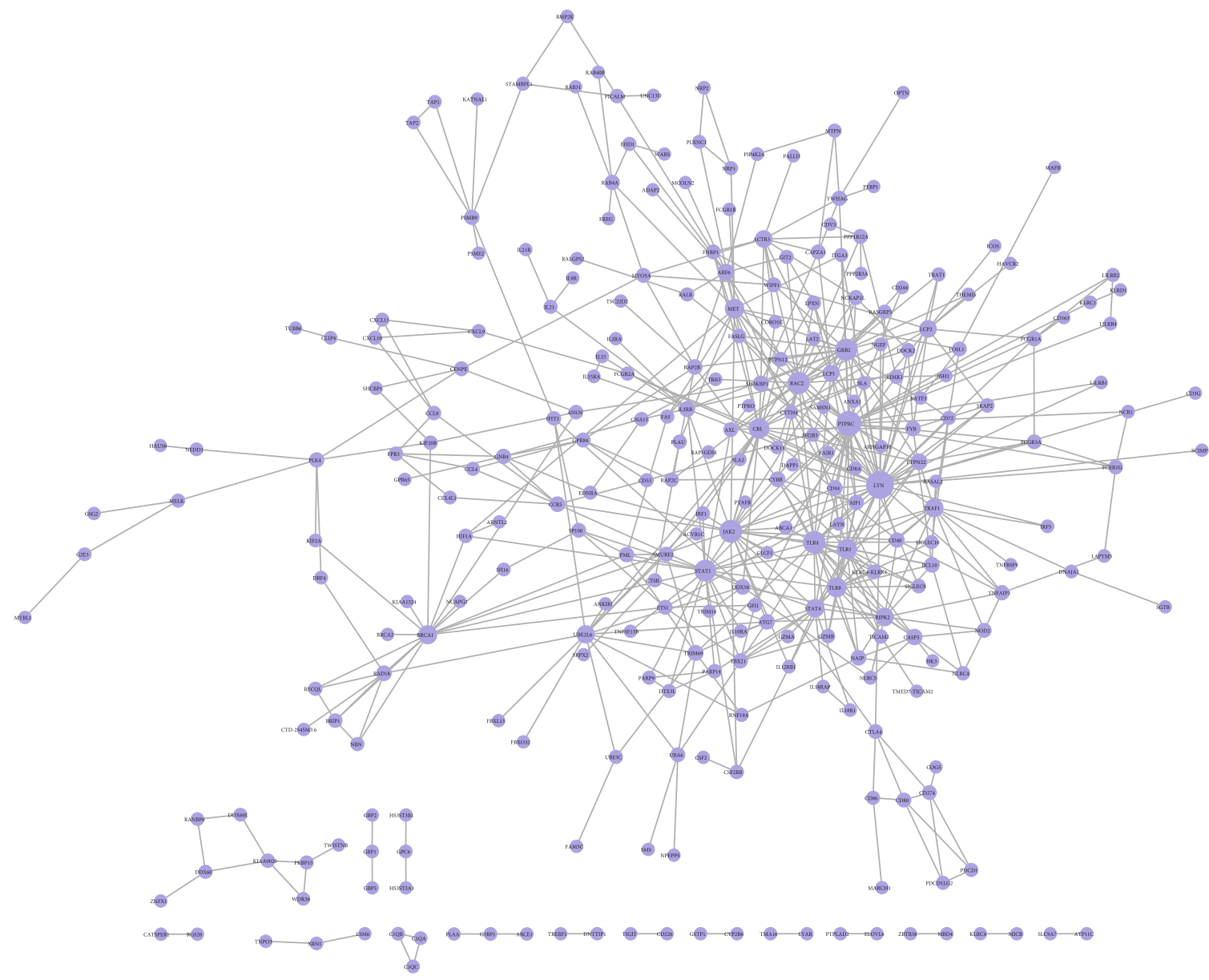

Figure 2: The map represents the protein-protein interaction network of PDL1 coexpressed genes. Nodes represent genes and lines connecting genes represent interactions.

Thirteen genes were excluded without the consistency of significant GO function and signaling pathway, four genes were excluded with no interaction in the PPI network. They play a role in immune response, response to stress, and so on. A mRNA-GO network including this group of genes was shown in Figure 5.

\section{Discussion}

Nowadays, the predictive biomarkers for PD1 and PDL1 immune checkpoint blockade therapy are a hot topic. Predictive biomarkers for treatment selection can improve efficiency and reduce costs while protecting patients from hazards of unnecessary treatment [14]. However, there is an unmet need for biomarkers that will identify patients more likely to respond to PD1/PDL1 blockade as well as other immunotherapeutics [15]. The PDL1 expression in combination with other potential biomarkers might get a higher predictive value for response to immune checkpoint inhibitors than the use of an individual biomarker.

In the present study, we sought to identify PDL1-related genes that contribute to the selection of lung adenocarcinoma patients for PD1/PDL1 inhibitor treatment in TCGA database. Treatment details of this set of samples were not available, so the samples diagnosed before April 22, 2014 was screened. Therefore, the patient had a small chance receiving PD1/PDL1 inhibitor therapy, which is a factor we need to consider in identifying candidate biomarkers. First, we identified 3 differential expression genes, EREG, BRCA1, and BRIP1, by comparing median survival time of the identified biomarkers and PDL1, constructing the coexpressed gene PPI network, ensuring consistency between function and signaling pathway, and searching progress in clinical application. Then, to find potential adjuvant therapeutic targets in immune checkpoint treatment, we performed transcription factor prediction analysis and focused on the nondifferentially expressed genes. With further exploration and validation, this observation might contribute to the lung adenocarcinoma patient selection for PD1/PDL1inhibitor therapy.

BRCA1 plays an important role in DNA repair and maintaining genomic stability, and it can also acts as tumor suppressor. BRIP1 is a member of the DEAH helicase family and it binds directly to the BRCT repeats of BRCA1. BRIP1 is a physiological partner of BRCA1, and BRIP1/BRCA1 complex formation contributed to the key activity of BRCA1 [16]. BRCA1 is wildly studied in NSCLC because of its role in chemotherapy response. Taron et al. revealed that BRCA1 


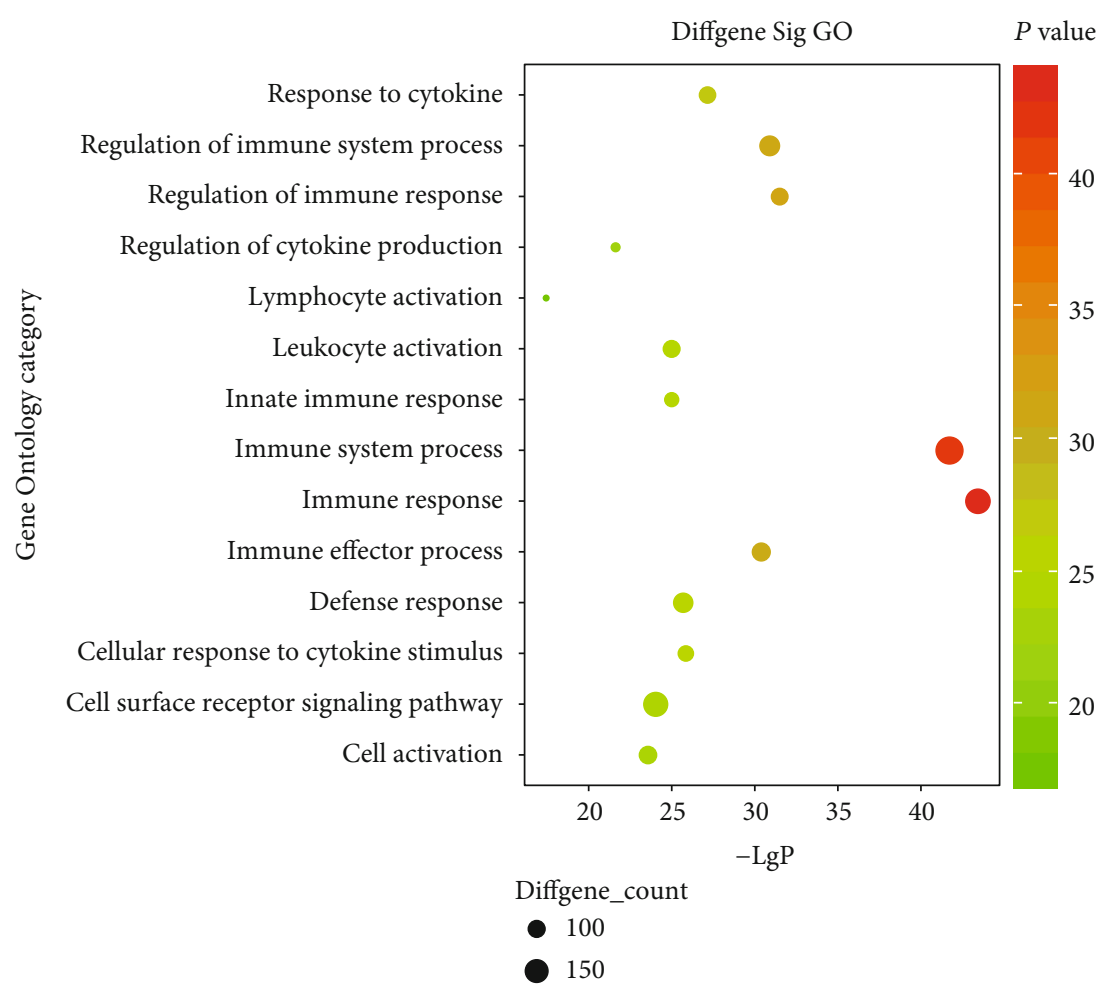

(a)

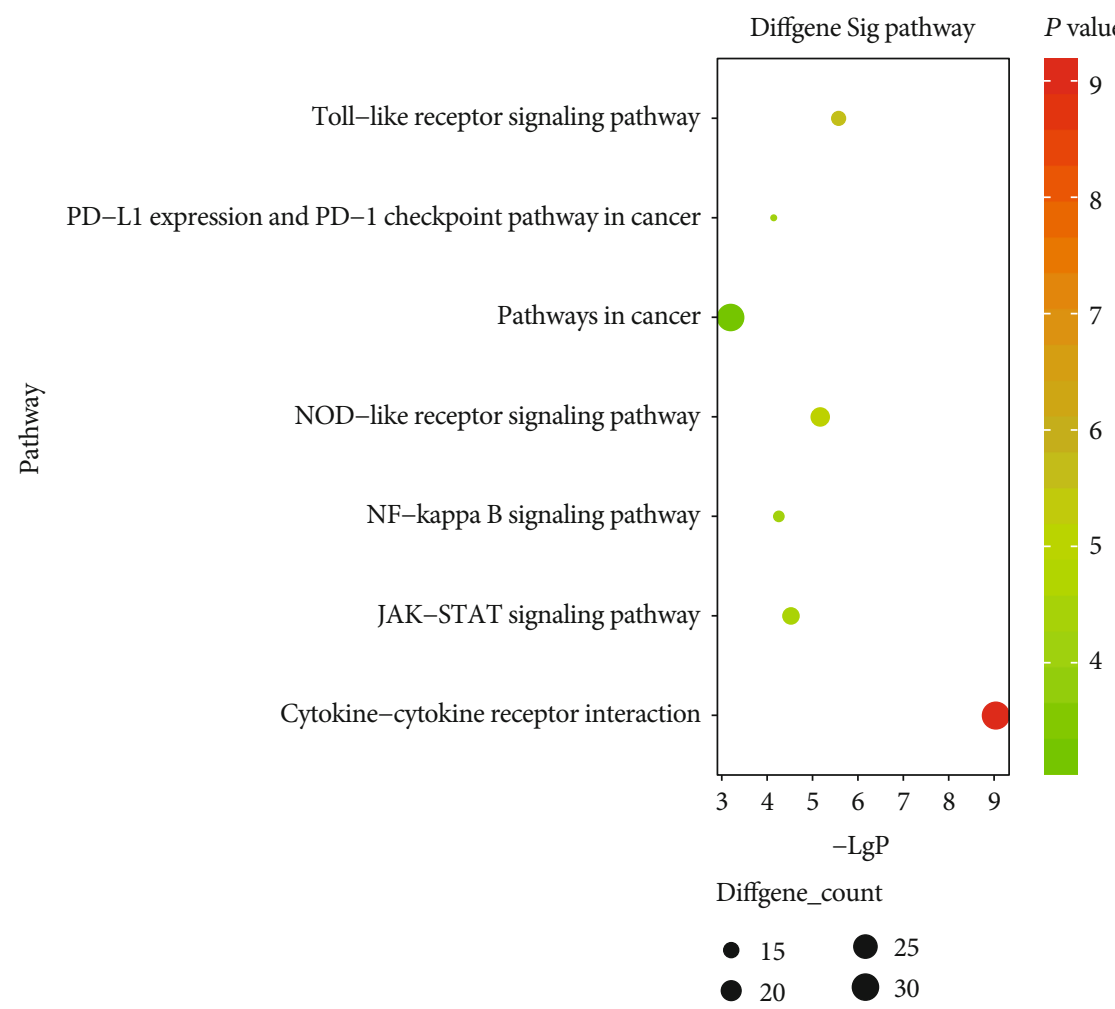

(b)

FIgURE 3: Top 14 enrichment of GO terms and top 7 enrichment of KEGG pathways for 39 candidate genes.

could be a predictor for differential chemosensitivity and personal chemotherapy in lung cancer $[17,18]$. It was pointed out that no statistically significant correlation was found in
BRCA1 expression in NSCLC regarding gender, age, histological type, or smoking status [19-21]. A study by Joerger et al. found that BRCA1 expression differs regarding gender 


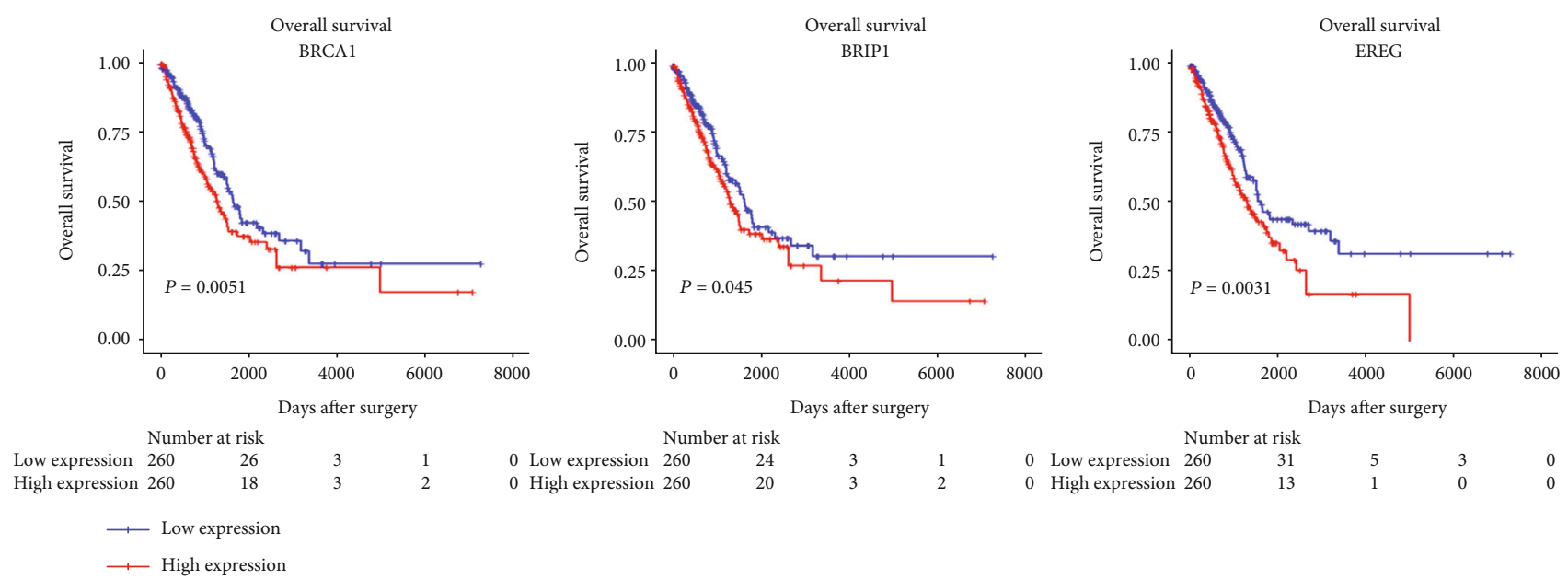

FIGURE 4: Three candidate genes of significant prognostic value. Kaplan-Meier curves showing the relationship between the three mRNAs and overall survival. The cases were divided into the low- and high-expression groups by the median expression level of genes.

TABLE 2: Top 10 transcription factor predictions of candidate genes.

\begin{tabular}{|c|c|c|c|c|c|}
\hline \multicolumn{2}{|r|}{ BRCA1 } & \multicolumn{2}{|r|}{ BRIP1 } & \multicolumn{2}{|r|}{ EREG } \\
\hline $\mathrm{TF}$ & Motif & $\mathrm{TF}$ & Motif & $\mathrm{TF}$ & Motif \\
\hline RFX4 & GGTTTCCGTGGCAACG & NFYB & AGCTCGACCAATCAC & E2F6 & GGGCGGGAGCA \\
\hline RFX5 & CGTTGCCACGGAAACC & TFDP1 & GGGCGGGAGGC & RXRG & GAGTGCACGGGCAGGGCG \\
\hline RFX5 & GGTTTCCGTGGCAACG & CTCFL & GTCGAGGGGGCGGG & RARA & AGTGCACGGGCAGGGCG \\
\hline RFX4 & CGTTGCCACGGAAACC & HAND1 & GGACTGGGGC & E2F6 & GGGAGGGAGGA \\
\hline RFX2 & CGTTGCCACGGAAACC & SP2 & TTCCCGCCTCCCGCC & NR2F1 & GTGAGGTCAAGAG \\
\hline RFX2 & GGTTTCCGTGGCAACG & CTCFL & GGACTGGGGCCGCC & NFYB & TCCCCGGCCAATCGG \\
\hline SOX10 & CGGACAAAGAC & SP2 & CTCCCGCCCCCTCGA & MEIS2 & CTGACAGC \\
\hline SOX3 & TCTTTGTCCG & TEAD2 & CCCCAGTCCTGCA & RUNX1 & CGCTGGGGCTT \\
\hline SOX6 & TCTTTGTCCG & TFDP1 & AGGCGGGAATT & $\mathrm{E} 2 \mathrm{~F} 4$ & GGGCGGGAGCA \\
\hline TFDP1 & GCGCGGGAATT & E2F6 & GGGCGGGAGGC & MAFG & GATGAC \\
\hline
\end{tabular}

TF, transcription factor; motif, DNA sequence of target species which is predicted to be bound by the binding protein.

in advanced NSCLC, but not age, histological type, pathological stage, or smoking status [22]. Results also showed that BRCA1 correlated with survival due to its role in chemotherapy response. Recently, there are a variety of clinical trials focusing on BRCA1 as a biomarker to provide prognostic information in NSCLC, but conflicting data requires further prospective validation and patient validation in clinical trials, also suggesting the importance of epigenetic BRCA1 expression [23-25]. As for BRIP1, Rosenthal et al. found that the variant is associated with the risk of colorectal cancer [26]. It was reported that elevated expression level is associated with increased metastasis and shortened survival in LUAD patients $[27,28]$. So far, limited results disclosed that the BRIP1 signature was prognostic for tumor stage, grade, and metastasis. Hence, BRCA1 and BRIP1 could be associated biomarkers for the PDL1-positive expression patient selection receiving chemotherapy and immune checkpoint inhibitor combination therapy needs further exploration and validation.

EREG is a member of the epidermal growth factor (EGF) family of peptide growth factors. Deregulated EREG activity contributes to the progression of a variety of malignancies, including non-small-cell lung cancer [29]. Researchers found that NSCLC with KRAS, BRAF, or EGFR mutations overexpressed EREG, and abrogation of such mutations or associated therapeutic inhibitors could downregulate the EREG expression [30]. Studies demonstrated that cancers, including NSCLC, with a higher tumor mutation burden, have a higher likelihood response to PD1/PDL1 blockades [31, 32]. A phase III trial demonstrated that EREG expression can be a predictor for overall survival in oxaliplatin/fluoropyrimidine plus bevacizumab-treated metastatic colorectal cancer patients without RAS and BRAF mutations [33]. High EREG and AREG expressions are a predictive marker for panitumumab therapy benefit on PFS in RAS wild-type advanced colorectal cancer patients [34]. It was reported that EREGhigh tumors in lung adenocarcinoma patients have significantly shorter DFS and OS compared to those with EREGlow tumors. EREG expression was associated with age, gender, and smoking status $[29,35]$. Elevated EREG expression was also related to pleural involvement positivity, lymphatic permeation positivity, and vascular invasion positivity. 


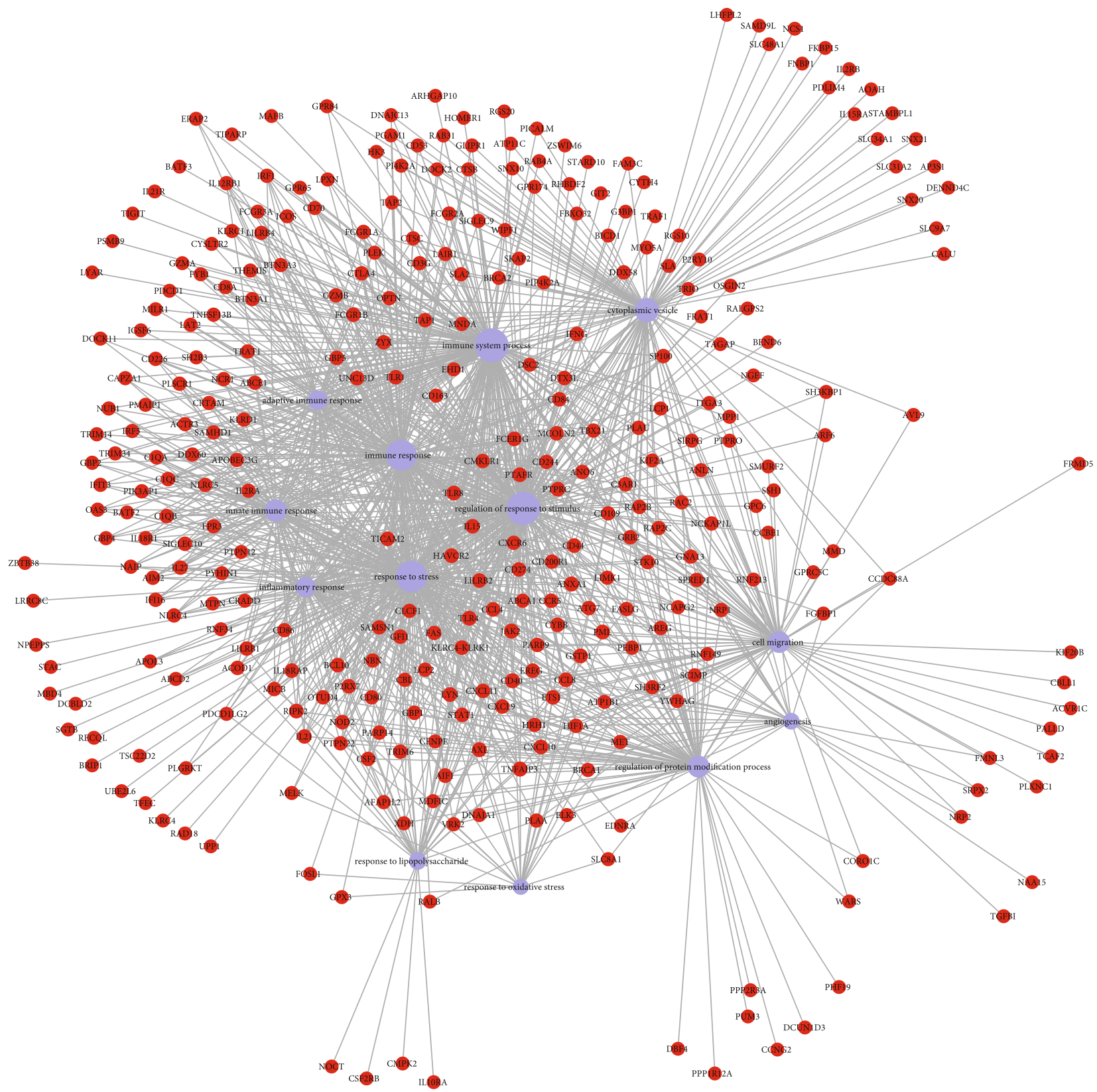

Figure 5: A mRNA-GO network mainly focus on thirteen GO terms. Blue nodes represent GO terms, red nodes represent genes, and lines represent interactions.

Therefore, EREG might be a predictive response biomarker for PDL1-positive expression patients receiving PD1/PDL1 inhibitors, which needs further exploration and validation.

We also conducted transcription factor analysis on these genes and tried to find potential adjuvant therapeutic targets for immune checkpoint inhibitor treatment. Transcription factors can directly bind to specific DNA tracts in gene promoters, which is a key step toward transcriptional activation or repression [36]. Studies revealed that transcription factors can be potential therapeutic targets based on its significance in a variety of biological processes and aberrant activity in human diseases [37]. A number of researches demonstrated that molecules aimed at targeting transcription factors is a promising strategy in cancer treatment $[38,39]$. We speculate that some transcription factors we predicted contribute to these differentially expressed genes we identified and might be potential adjuvant therapeutic targets for PD1 and PDL1 immune checkpoint blockade therapy in lung adenocarcinoma. Future work needs to be done to identify the main transcription factor that regulates the gene expression.

Finally, to identify adjuvant biomarkers that is PDL1 related but negative differential expression genes. Although the expression of these genes has no difference compared with the control, but high or low expression has a prognostic value. CCR5 is expressed higher in invasive tumor tissues than noninvasive tissues according to the subclassification of adenocarcinoma [40]. CCR5 can be expressed by several cell types and there is an elevated CCR5 MDSC expression 
level in patients with NSCLC [41]. Some evidence suggests that CCR5 and its ligands appear to participate in the canonical immune checkpoint response and elevated expression level correlated with a poor prognosis [42]. It was reported that KRAS can regulate FOSL1 expression and it was an independent survival marker for LADC patients with KRAS mutations [43]. And low expression of FOSL1 in the cytoplasm was linked with advanced tumor stage [44]. NAIP is an antiapoptotic protein and the research in NSCLC is limited. NBN is regarded to be involved in DNA double-strand break repair and DNA damage-induced checkpoint activation. There is a report about NBN mutation in lung cancer [45]. NBN polymorphisms may be genetic biomarkers for NSCLC prognosis especially PFS with platinum-based chemotherapy in the Chinese population [46]. Whether this group of biomarkers could help with the patient selection needs further research. And the expression and the function of these biomarkers in NSCLC need further validation and description in the future. At the same time, cellular sublocalization, mutation, and epigenetic modification should be paid attention to.

As we mentioned before, the PDL1 immunohistochemistry evaluation was not available and the mRNA expression level was used to predict the survival time. In this study, we found that median survival time of patients in the PDL1 high-expression group was 701 days. Median survival time in high expression of the identified biomarkers, BRCA1, BRIP1, and EREG, was 690, 701, and 690 days separately. As for median survival time, biomarkers we identified were not superior to PDL1. It might attribute to the limited sample size and needs further large sample validation. Another reason might be because we assess mRNA levels not protein expression levels; future exploration and validation need to focus on protein expression. There are some limitations using immunohistochemistry to detect the candidate biomarkers we identified. First, expression of BRCA1, BRIP1, and EREG was regulated by various mechanisms, such as signaling pathway, transcription factors as we predicted, and epigenetic factors. Individual difference and intratumor heterogeneity should be taken into account. Second, poor uniformity in immunohistochemistry antibodies and different score algorithms may cause the inaccuracy. So, in the future, large-scale investigation and validation need to be done clinically, and new detection methods need to be developed.

\section{Conclusions}

In conclusion, from the functional enrichment analysis of TCGA database, we extracted a list of PDL1-related genes. These genes have the potential to become additional biomarkers for the selection of patients for PD1/PDL1 inhibitors. The predicted transcription factors of these genes might provide adjuvant therapeutic targets and improve the immune therapy responsiveness. In addition, it would be extremely meaningful if the combination of PDL1 and these potential biomarkers have a more accurate indication of patient selection. Further investigation of these genes could lead to novel insights into the immunotherapy for lung adenocarcinoma patients.

\section{Data Availability}

The data used to support the findings of this study are included within the article.

\section{Conflicts of Interest}

The authors declare that they have no conflicts of interest.

\section{Acknowledgments}

This work was supported by the National Natural Science Foundation of China (grant number 81670043).

\section{References}

[1] L. A. Torre, F. Bray, R. L. Siegel, J. Ferlay, J. Lortet-Tieulent, and A. Jemal, "Global cancer statistics, 2012," CA: a Cancer Journal for Clinicians, vol. 65, no. 2, pp. 87-108, 2015.

[2] T. B. Richards, S. J. Henley, M. C. Puckett et al., "Lung cancer survival in the United States by race and stage (2001-2009): findings from the CONCORD-2 study," Cancer, vol. 123, Supplement 24, pp. 5079-5099, 2017.

[3] W. D. Travis, E. Brambilla, A. G. Nicholson et al., "The 2015 World Health Organization classification of lung tumors," Journal of Thoracic Oncology, vol. 10, no. 9, pp. 1243-1260, 2015.

[4] M. McNutt, "Cancer immunotherapy," Science, vol. 342, no. 6165 , article 1417, 2013.

[5] S. J. Antonia, A. Villegas, D. Daniel et al., "Overall survival with durvalumab after chemoradiotherapy in stage III NSCLC," The New England Journal of Medicine, vol. 379, no. 24, pp. 2342-2350, 2018.

[6] F. Yaqub, "Nivolumab for squamous-cell non-small-cell lung cancer," The Lancet Oncology, vol. 16, no. 7, article e319, 2015.

[7] T. S. K. Mok, Y. L. Wu, I. Kudaba et al., "Pembrolizumab versus chemotherapy for previously untreated, PD-L1-expressing, locally advanced or metastatic non-small-cell lung cancer (KEYNOTE-042): a randomised, open-label, controlled, phase 3 trial," Lancet, vol. 393, no. 10183, pp. 1819-1830, 2019.

[8] H. West, M. McCleod, M. Hussein et al., "Atezolizumab in combination with carboplatin plus nab-paclitaxel chemotherapy compared with chemotherapy alone as first-line treatment for metastatic non-squamous non-small-cell lung cancer (IMpower130): a multicentre, randomised, open-label, phase 3 trial," The Lancet Oncology, vol. 20, no. 7, pp. 924-937, 2019.

[9] "FDA approves durvalumab after chemoradiation for unresectable stage III NSCLC," http://www.fda.gov/drugs/ resources-information-approved-drugs/durvalumab-imfinzi.

[10] P. N. Aguiar Jr., I. L. Santoro, H. Tadokoro et al., “The role of $\mathrm{PD}-\mathrm{L} 1$ expression as a predictive biomarker in advanced nonsmall-cell lung cancer: a network meta-analysis," Immunotherapy, vol. 8, no. 4, pp. 479-488, 2016.

[11] X. Meng, Z. Huang, F. Teng, L. Xing, and J. Yu, "Predictive biomarkers in PD-1/PD-L1 checkpoint blockade immunotherapy," Cancer Treatment Reviews, vol. 41, no. 10, pp. 868876, 2015.

[12] J. McLaughlin, G. Han, K. A. Schalper et al., "Quantitative assessment of the heterogeneity of PD-L1 expression in nonsmall-cell lung Cancer," JAMA Oncology, vol. 2, no. 1, pp. 46-54, 2016. 
[13] M. I. Love, W. Huber, and S. Anders, "Moderated estimation of fold change and dispersion for RNA-seq data with DESeq2," Genome Biology, vol. 15, no. 12, p. 550, 2014.

[14] G. Dranitsaris, X. Zhu, G. Adunlin, and M. D. Vincent, "Cost effectiveness vs. affordability in the age of immuno-oncology cancer drugs," Expert Review of Pharmacoeconomics \& Outcomes Research, vol. 18, no. 4, pp. 351-357, 2018.

[15] S. L. Topalian, J. M. Taube, R. A. Anders, and D. M. Pardoll, "Mechanism-driven biomarkers to guide immune checkpoint blockade in cancer therapy," Nature Reviews. Cancer, vol. 16, no. 5, pp. 275-287, 2016.

[16] S. B. Cantor, D. W. Bell, S. Ganesan et al., "BACH1, a novel helicase-like protein, interacts directly with BRCA1 and contributes to its DNA repair function," Cell, vol. 105, no. 1, pp. 149-160, 2001.

[17] M. Taron, R. Rosell, E. Felip et al., "BRCA1 mRNA expression levels as an indicator of chemoresistance in lung cancer," Human Molecular Genetics, vol. 13, no. 20, pp. 2443-2449, 2004.

[18] G. Bepler, M. Begum, and G. R. Simon, "Molecular analysisbased treatment strategies for non-small cell lung cancer," Cancer Control, vol. 15, no. 2, pp. 130-139, 2008.

[19] A. Lafuente-Sanchis, Á. Zúñiga, J. M. Galbis et al., "Prognostic value of ERCC1, RRM1, BRCA1 and SETDB1 in early stage of non-small cell lung cancer," Clinical \& Translational Oncology, vol. 18, no. 8, pp. 798-804, 2016.

[20] I. Boukovinas, C. Papadaki, P. Mendez et al., “Tumor BRCA1, RRM1 and RRM2 mRNA expression levels and clinical response to first-line gemcitabine plus docetaxel in nonsmall-cell lung Cancer patients," PLoS One, vol. 3, no. 11, article e3695, 2008.

[21] T. Fujii, S. Toyooka, K. Ichimura et al., "ERCC1 protein expression predicts the response of cisplatin-based neoadjuvant chemotherapy in non-small-cell lung cancer," Lung Cancer, vol. 59, no. 3, pp. 377-384, 2008.

[22] M. Joerger, D. deJong, A. Burylo et al., "Tubuline, BRCA1, ERCC1, Abraxas, RAP80 mRNA expression, p 53/p 21 immunohistochemistry and clinical outcome in patients with advanced non small-cell lung cancer receiving first-line platinum-gemcitabine chemotherapy," Lung Cancer, vol. 74, no. 2, pp. 310-317, 2011.

[23] G. Levallet, F. Dubois, P. Fouret et al., "MSH2/BRCA1 expression as a DNA-repair signature predicting survival in earlystage lung cancer patients from the IFCT-0002 Phase 3 Trial," Oncotarget, vol. 8, no. 3, pp. 4313-4329, 2017.

[24] R. Rosell, M. Skrzypski, E. Jassem et al., "BRCA1: a novel prognostic factor in resected non-small-cell lung cancer," PLOS One, vol. 2, no. 11, article e1129, 2007.

[25] W. E. Pierceall, K. A. Olaussen, V. Rousseau et al., "Cisplatin benefit is predicted by immunohistochemical analysis of DNA repair proteins in squamous cell carcinoma but not adenocarcinoma: theranostic modeling by NSCLC constituent histological subclasses," Annals of Oncology, vol. 23, no. 9, pp. 2245-2252, 2012.

[26] E. A. Rosenthal, NHLBI GO Exome Sequencing Project, B. H. Shirts et al., "Rare loss of function variants in candidate genes and risk of colorectal cancer," Human Genetics, vol. 137, no. 10 , pp. 795-806, 2018.

[27] L. Lignitto, S. E. LeBoeuf, H. Homer et al., "Nrf2 Activation Promotes Lung Cancer Metastasis by Inhibiting the Degradation of Bach1," Cell, vol. 178, no. 2, pp. 316-329.e18, 2019.
[28] The Cancer Genome Atlas Research Network, "Comprehensive molecular profiling of lung adenocarcinoma," Nature, vol. 511, no. 7511, pp. 543-550, 2014.

[29] N. Sunaga, K. Kaira, H. Imai et al., “Oncogenic KRAS-induced epiregulin overexpression contributes to aggressive phenotype and is a promising therapeutic target in non-small-cell lung cancer," Oncogene, vol. 32, no. 34, pp. 4034-4042, 2013.

[30] N. Fujimoto, M. Wislez, J. Zhang et al., "High expression of ErbB family members and their ligands in lung adenocarcinomas that are sensitive to inhibition of epidermal growth factor receptor," Cancer Research, vol. 65, no. 24, pp. 11478 11485, 2005.

[31] L. B. Alexandrov, Australian Pancreatic Cancer Genome Initiative, S. Nik-Zainal et al., "Signatures of mutational processes in human cancer," Nature, vol. 500, no. 7463, pp. 415-421, 2013.

[32] A. M. Goodman, S. Kato, L. Bazhenova et al., "Tumor mutational burden as an independent predictor of response to immunotherapy in diverse cancers," Molecular Cancer Therapeutics, vol. 16, no. 11, pp. 2598-2608, 2017.

[33] S. Stintzing, B. Ivanova, I. Ricard et al., “Amphiregulin (AREG) and epiregulin (EREG) gene expression as predictor for overall survival (OS) in oxaliplatin/fluoropyrimidine plus bevacizumab treated mCRC patients-analysis of the phase III AIO KRK-0207 trial," Frontiers in Oncology, vol. 8, p. 474, 2018.

[34] J. F. Seligmann, F. Elliott, S. D. Richman et al., "Combined epiregulin and amphiregulin expression levels as a predictive biomarker for panitumumab therapy benefit or lack of benefit in patients with RAS wild-type advanced colorectal cancer," JAMA Oncology, vol. 2, no. 5, pp. 633-642, 2016.

[35] J. Zhang, K. Iwanaga, K. C. Choi et al., "Intratumoral epiregulin is a marker of advanced disease in non-small cell lung cancer patients and confers invasive properties on EGFR-mutant cells," Cancer Prevention Research (Philadelphia, PA), vol. 1, no. 3, pp. 201-207, 2008.

[36] J. M. Vaquerizas, S. K. Kummerfeld, S. A. Teichmann, and N. M. Luscombe, "A census of human transcription factors: function, expression and evolution," Nature Reviews. Genetics, vol. 10, no. 4, pp. 252-263, 2009.

[37] K. A. Papavassiliou and A. G. Papavassiliou, "Transcription factor drug targets," Journal of Cellular Biochemistry, vol. 117, no. 12, pp. 2693-2696, 2016.

[38] C. Rajagopal, M. B. Lankadasari, J. M. Aranjani, and K. B. Harikumar, "Targeting oncogenic transcription factors by polyphenols: a novel approach for cancer therapy," Pharmacological Research, vol. 130, pp. 273-291, 2018.

[39] J. E. Yeh, P. A. Toniolo, and D. A. Frank, "Targeting transcription factors: promising new strategies for cancer therapy," Current Opinion in Oncology, vol. 25, no. 6, pp. 652658, 2013.

[40] A. C. Borczuk, N. Papanikolaou, R. L. Toonkel et al., "Lung adenocarcinoma invasion in TGFbetaRII-deficient cells is mediated by CCL5/RANTES," Oncogene, vol. 27, no. 4, pp. 557-564, 2008.

[41] Y. Yamauchi, S. Safi, C. Blattner et al., "Circulating and tumor myeloid-derived suppressor cells in resectable non-small cell lung cancer," American Journal of Respiratory and Critical Care Medicine, vol. 198, no. 6, pp. 777-787, 2018.

[42] X. Jiao, O. Nawab, T. Patel et al., "Recent advances targeting CCR5 for cancer and its role in immuno-oncology," Cancer Research, vol. 79, no. 19, pp. 4801-4807, 2019. 
[43] A. Vallejo, N. Perurena, E. Guruceaga et al., "An integrative approach unveils FOSL1 as an oncogene vulnerability in KRAS- driven lung and pancreatic cancer," Nature Communications, vol. 8, no. 1, article 14294, 2017.

[44] K. Ma, D. Chang, M. Gong et al., "Expression and significance of FRA-1 in non-small-cell lung cancer," Cancer Investigation, vol. 27, no. 3, pp. 353-359, 2009.

[45] C. L. Chuang, C. H. Wang, C. H. Hsu et al., "Contribution of double-strand break repair gene Nijmegen breakage syndrome 1 genotypes, gender difference and smoking status to Taiwanese lung cancer," Anticancer Research, vol. 37, no. 5, pp. 24172423, 2017.

[46] J. L. Xu, L. M. Hu, M. D. Huang et al., "Genetic variants of NBS1 predict clinical outcome of platinum-based chemotherapy in advanced non-small cell lung cancer in Chinese," Asian Pacific Journal of Cancer Prevention, vol. 13, no. 3, pp. 851856, 2012. 